\title{
MFDTool: A software program for designing optimal multifunction displays
}

\author{
GREGORY FRANCIS \\ Purdue University, West Lafayette, Indiana
}

\begin{abstract}
This paper describes a software program called MFDTool. MFDTool is used to design multifunction displays (MFDs), which enable people to interact with computer information systems. MFDTool characterizes the problem of MFD design as the identification of how people interact with the system and what types of constraints should be imposed by the designer to shape the properties of the MFD. Once this information is provided, MFDTool uses an optimization procedure to build a variety of candidate MFD designs and to identify the MFD design that best satisfies the constraints. An example of MFD design is discussed.
\end{abstract}

As computers are integrated into more devices, the human factors issues of interfacing with the computer become ever more important. The design of computer interfaces has been shown to be related to the effective use and learning and the tendency to make errors with a variety of systems. The design has been shown to affect user performance in a variety of devices, including automated tellers (Rogers, Cabrera, Walker, Gilbert, \& Fisk, 1996), medical devices (Cook \& Woods, 1996; Obradovich \& Woods, 1996), Intranet information servers (Cuomo, Borghesani, Khan, \& Violett, 1998), and aircraft flight controls (Reising \& Curry, 1987; Sirevaag et al., 1993).

This paper focuses on a special type of humancomputer interface known as a multifunction display (MFD). MFDs involve fixed hardware switches and flexible software pages. The information in these devices is often (but not always) arranged hierarchically so that a user starts at a top level and moves down the hierarchy by selecting appropriate MFD pages. Often, the hardware devices are real or simulated buttons that remain in fixed positions. Common examples of MFDs include automated teller machines, pagers, aircraft cockpit display panels, and various medical devices. The creation of effective

This work was supported in part by the Army Aeromedical Research Laboratory (Dr. Kent A. Kimball) under the auspices of the U.S. Army Research Office Scientific Services Program administered by Battelle (Delivery Order 1832, Contract No. DAAL0391C0034; Delivery Order 63, Contract No. DAAH0496C0086; and Delivery Order 224, Contract No. DAAH0496C0086). The views, opinions, and/or findings contained in this report are those of the author and should not be construed as an official Department of the Army position, policy, or decision, unless so designated by other documentation. The author retains a propretary interest in further developments of the MFDTool software. Correspondence concerning this article should be addressed to G. Francis, Department of Psychological Sciences, Purdue University, 703 Third Street, West Lafayette, IN 47907-2004 (e-mail: gfrancis@ psych. purdue.edu).
MFDs is a difficult task (Francis, 2000; Reardon \& Francis, 1999; Williges, Williges, \& Fainter, 1988). Designers of MFDs must decide how many buttons to include, the hierarchical arrangement of information, and the mapping of page labels to hardware (typically, buttons).

One of the findings of human factors research is that the specific details of the human-computer system have a significant impact on determining the appropriate design. This means that previously created MFD designs offer only partial guidance for the development of new MFD designs. The underlying principles that guided the design of one MFD system may not apply to another.

MFDTool is a computer program that focuses on a particular subtask of MFD design-namely, how page labels in an information hierarchy should be assigned to hardware buttons. Mapping hierarchy information to MFD buttons is a challenging task. The human-computer interactions involved in accessing information from an MFD are complicated and not entirely understood. Therefore, such mappings are, at best, created by experts who rely on experience and general guidelines (Calhoun, 1978; Department of Defense, 1981; Holley \& Busbridge, 1995; Lind, 1981; Spiger \& Farrell, 1982; Williges et al., 1988).

Although design experience is certainly valuable and general guidelines can help the design process, there are limits to these approaches. Even a small hierarchy database can be mapped to hardware buttons in a vast number of ways (Fisher, Yungkurth, \& Moss, 1990), so combinatorial explosion quickly precludes an exhaustive search of all possible mappings. As a result, even experienced designers are unable to ensure that the guidelines that they believe are important for their particular design task are satisfied.

The MFDTool program requires that the designer know the properties of the MFD hardware and the properties of the users who will use the system. MFDTool then guides the creation of a model of the various ways the users will 
interact with the computer. These interactions then form the basis for a variety of constraints that the designer believes should be satisfied by the design of the MFD. In essence, MFDTool provides a methodology by which to quantify the guidelines so that an optimization can be performed to construct the best MFD design.

In addition to providing immediate practical benefit to interface designers, MFDTool should be useful to academics who want to understand the underlying mechanisms that contribute to good and poor interface designs. For example, if a researcher believes that a voice recognition interface is better than a push-button interface, a fair comparison needs to be made between the best possible designs for both interface types (Reising \& Curry, 1987). MFDTool should speed the design of optimal interfaces and, thereby, allow comparisons of different modalities. Likewise, if a researcher believes that design consistency is important (e.g., always have a "Cancel" button on the bottom left), one needs to compare a design that is optimized but has that consistency against one that is optimized without that type of consistency. Any other comparison of designs does not really measure the full impact of consistency. Current approaches to MFD design are so labor intensive that investigation of the factors involved in human-computer interaction of MFDs has been difficult. MFDTool provides a means of producing optimized types of designs and, so, should allow for careful study of the importance of different guidelines for interface design.

The goal of this paper is not to describe the inner workings of MFDTool but to describe the flavor of the system's approach to MFD design. A full discussion of the program itself, and of its own interface, windows, and parameters is lengthy and is given in detail in a user's guide that is available with the MFDTool program. A demonstration version of MFDTool can be downloaded on the Internet from http://www.psych.purdue.edu/ gfrancis/MFDTool. MFDTool is written in the Java programming language, so it will run on virtually any modern computer.

\section{MFDTool Design Analysis}

MFDTool approaches the design process as the integration of several types of information. These include hardware information, user information, interactions, and designer constraints. The way these information sources conceptually contribute to the design task is schematized in Figure 1.

Hardware information refers to a variety of details about the physical properties of the MFD. In many cases, this information will include details about the size of the display, the number of labels that can be shown on a given screen, the space between buttons (if buttons are used), the size of the buttons, and so forth. MFDTool requires that much of this information be given explicitly to the program. This information is used to define interactions, as will be described below. In some cases, there may be multiple types of hardware information, because there may be multiple means of interfacing with a database of information.

User information refers to details about the properties of the individuals who will interact with the MFD. This information might include whether an individual will have lots of experience with the MFD or will be a first-time user. It might also include information about the visual acuity of the user, the manual dexterity of the user, the memory abilities of the user, and so forth. There may be a large number of users with very different characteristics, and each type of user may need to be considered separately.

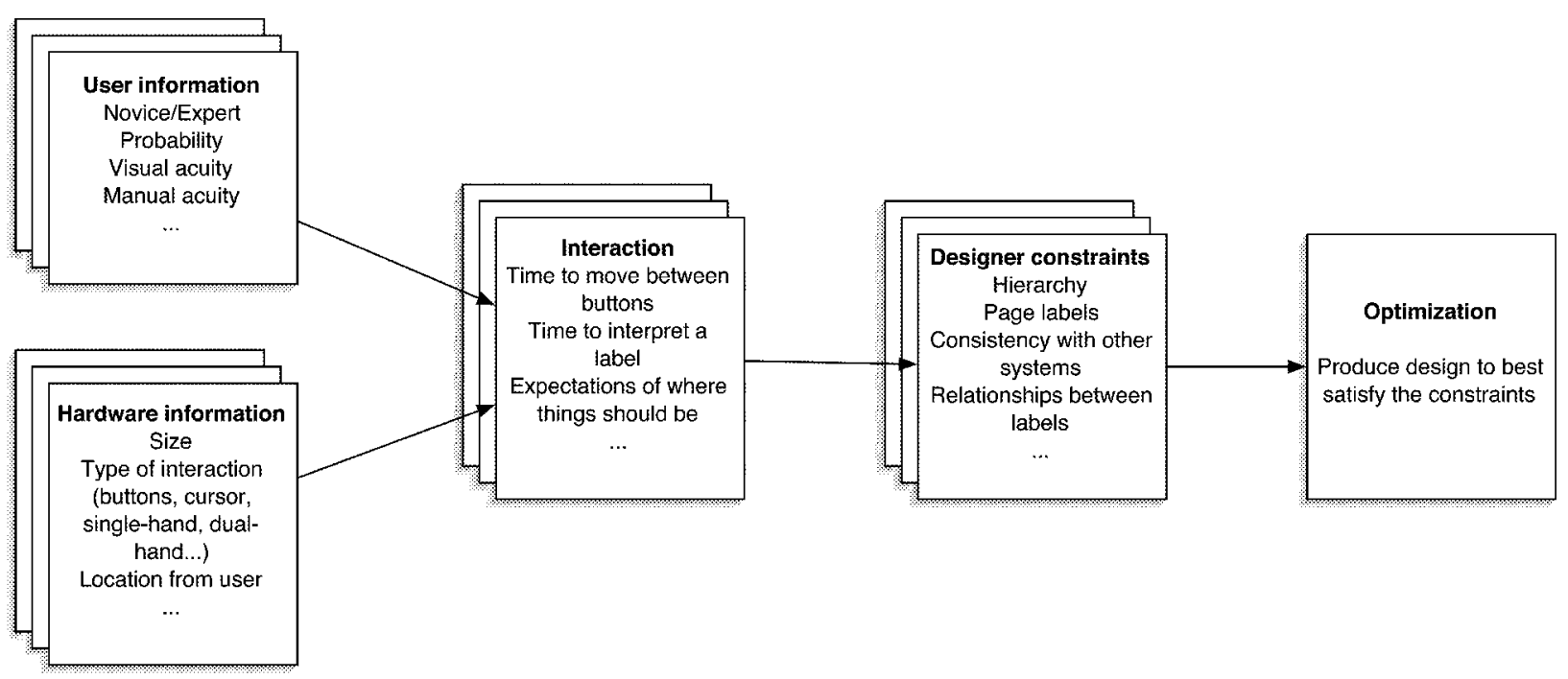

Figure 1. A diagram of the factors contributing to multifunction display (MFD) design with MFDTool. MFDTool integrates MFD hardware properties and user properties to create interactions. The designer imposes constraints on the interactions to provide relationships between the labels and the buttons. MFDTool then uses standard optimization techniques to find the MFD design that best satisfies the constraints. 
None of this information is explicitly given to the MFDTool program. Instead, the user information contributes to the definition of various types of interactions.

An interaction refers to a way in which the user information and hardware information affect the ability of the user to work with the MFD. Although an MFD may be casually described as having one type of human-computer interaction (e.g., pressing of bezel buttons), in reality, multiple interactions are involved in almost every system. For example, to press bezel buttons, a user needs to be able to see the buttons and reach them. Thus, both the visual display of information (a type of visual interaction) and the motor commands to reach buttons (a type of motor interaction) are involved. These different interactions have different properties, and the MFD design should consider those differences. As another example, in many instances, successful use of the MFD depends on the user's being able to remember where certain information is located. A memory interaction may be related to other types of interactions but may also introduce its own idiosyncrasies.

In addition to having multiple interactions, a single MFD might be used in multiple ways and by multiple users. Each combination of interface technique and user might require a new definition of an interaction. For example, in a military cockpit, a pilot may have multiple means of interacting with the MFD. Bezel buttons around the MFD are a common type of touch interaction. A handon-throttle control is often another means of interacting with the MFD. The MFD should be designed so that the pilot can quickly and efficiently use either type of interaction to retrieve information from the MFD. In addition, a copilot may also have multiple interactions, but the frequency with which the copilot uses the hand-on-throttle interaction may be lower than that for the pilot. An MFD designer should consider all of these factors and build an MFD that is optimized to accommodate all types of interactions by different people. Several examples of interactions are described below.

Constraints are imposed by the MFD designer to identify how the MFD should be constructed to make the human-computer interface the best it can be. For example, one constraint might be the request that labels with the same name but on different MFD pages should be placed in a common position. A different constraint might be the request that all of the "Cancel" buttons should be located on one of the two bottom positions of an MFD. Yet another constraint might be the request that the average time to reach information in an MFD should be minimized. Each constraint is associated with a particular interaction, and a single interaction might have multiple constraints. Likewise, similar types of constraints might exist for a variety of different interactions. Each constraint is quantitatively defined so that a cost function can be computed. Smaller cost functions mean that the constraint is better satisfied.

Once the interactions and constraints are defined, MFDTool uses standard optimization techniques to guide a search through a large number of candidate designs. For each candidate design, MFDTool computes the total con- straint cost. Because everything is defined quantitatively, MFDTool can consider many more designs than even the most experienced human designers. As it searches through candidate designs, MFDTool keeps the design that provides the lowest total constraint cost. This design is the optimal design for the interactions and constraints that were provided by the designer.

\section{Predefined Interactions}

The quality of the design solution found by MFDTool depends on the ability of the designer to identify the interactions and constraints that characterize the important aspects of the human-computer interface.

In MFDTool, each type of interaction is defined by a set of interaction coefficients, with each coefficient representing a relationship between a pair of labels. For example, a Euclidean distance interaction would consist of interaction coefficients that identify the physical distance between each pair of labels (or the buttons associated with labels). As another example, some MFDs have the user push arrow keys to "tab" through labels. The interaction coefficients here might indicate the number of pushes that are required to go from one label to another.

Interaction coefficients need not be true distances in the mathematical sense. For example, in some situations, it may be difficult to go from the left side of the MFD to the right side but easy to go the other way (e.g., perhaps the hand occludes the buttons and labels on the right side). Although it breaks down for some interactions, a designer can often think of an interaction coefficient as identifying how difficult it is for a user to go from one label to another. Larger numbers indicate more difficulty.

In MFDTool, a designer can specify the interaction coefficients to be whatever is desired. For an MFD design crafted for a particular individual, multiple interactions can be used to describe the way the user will interact with the MFD. One interaction might describe the movement time between buttons, taking into account the size of the user's hand, the reach of the user's fingers, and the speed of movements. Another interaction might describe the ability of the user to search for labels, taking into account aspects of the user's ability to identify targets in his/her peripheral vision. Still another interaction might describe an entirely different type of user interface (e.g., hand-onthrottle controls in military aircraft).

At the same time, an MFD may be used by multiple individuals. To satisfy all of these users, the designer could define separate sets of interactions for each individual user or could combine data across individuals and define interactions for different sets of users (e.g., small, medium, or large hands).

There is no denying that it may be a difficult task, in many instances, to specify the interaction coefficients for a particular MFD and particular users. The task requires an understanding of the human-computer interface and, perhaps, the creation of a model that describes how the user(s) will interact with the interface. To aid in the creation of interactions, MFDTool provides an easy means of 
calculating five sets of interaction coefficients that are computed from the physical properties of the MFD hardware. These will be described below.

\section{Fitts's Movement}

A common form of interaction with an MFD is fingerpointing, where a user presses buttons by moving his finger (or perhaps a mouse or pointer pen) from one button to another. There is a well-established model of how long it takes people to move a pointer over a given distance to a target of a given size. MFDTool uses a form of Fitts's Law (Fitts, 1954) that says that the movement time, $I[i, j]$, between button $i$ and button $j$ is

$$
I[i, j]=I_{m} \log _{2}\left(\frac{2 H_{i j}}{S_{j}}+1\right) .
$$

Here, $H_{i j}$ is the center-to-center Euclidean distance between the buttons, $S_{j}$ is the size of the second button (defined as the minimum of button height and width), $\log _{2}$ is the logarithm in base 2 , and $I_{m}$ is a parameter with units of milliseconds/bit. $I_{m}$ is empirically measured, and, for finger movements, values between 70 and $120 \mathrm{msec} / \mathrm{bit}$ are common. MFDTool uses $I_{m}=100 \mathrm{msec} / \mathrm{bit}$.

Constraints on this interaction can be set, for example, to minimize the time needed to move between buttons while retrieving information from the MFD. It is surely easier to press the same button four times than to move back and forth across the MFD screen to press a sequence of buttons. A designer may want to ensure that frequently used page labels are assigned to buttons so that reaching that page label involves repeated selection of a common button.

\section{Euclidean Distance}

This set of interaction coefficients describes the physical distance between button centers. The interaction coefficient for two buttons $i$ and $j$, centered on $\left(x_{i}, y_{i}\right)$ and $\left(x_{j}, y_{j}\right)$, respectively, is computed as

$$
I[i, j]=\sqrt{\left(x_{i}-x_{j}\right)^{2}+\left(y_{i}-y_{j}\right)^{2}} .
$$

This distance is useful whenever a physical relationship is called for. A designer may want to ensure that related labels are in physically similar locations. If the same type of page label is in a consistent location across different pages, this might allow for visual grouping and help the development of memory.

\section{City Block Distance}

This set of interaction coefficients describes the city block distance between button centers. The interaction coefficient for two buttons $i$ and $j$ centered on $\left(x_{i}, y_{i}\right)$ and $\left(x_{j}, y_{j}\right)$, respectively, is computed as

$$
I[i, j]=\left|x_{i}-x_{j}\right|+\left|y_{i}-y_{j}\right| .
$$

City block distance might be useful as an intermediate step in the calculation of some types of interactions. For example, an interface that involves tabbing through menu options may use an interaction that is a variation of a city block distance.

\section{$X$-Directed}

This set of interaction coefficients describes the horizontal distance and direction of button centers. The interaction coefficient for two buttons $i$ and $j$ centered on $\left(x_{i}, y_{i}\right)$ and $\left(x_{j}, y_{j}\right)$, respectively, is computed as

$$
I[i, j]=x_{i}-x_{j} \text {. }
$$

This coefficient does not really indicate difficulty of movement between a pair of buttons, but the absolute value of this coefficient would indicate the horizontal distance between buttons. $X$-directed is most useful for situations in which the designer wants to constrain directional relationships between labels. For example, a designer could specify that all labels named "Checking" should be on a button that is to the left of the previous buttonpress. This might introduce a type of motor memory that would make learning to use the MFD easier.

\section{$Y$-Directed}

This set of interaction coefficients describes the vertical distance and direction of button centers. The interaction coefficient for two buttons $i$ and $j$ centered on $\left(x_{i}, y_{i}\right)$ and $\left(x_{j}, y_{j}\right)$, respectively, is computed as

$$
I[i, j]=y_{i}-y_{j} \text {. }
$$

Position $(0,0)$ corresponds to the upper left corner of the MFD screen. A positive interaction coefficient indicates that the second button is above the first. A negative coefficient indicates that the second button is below the first. $Y$-directed has pretty much the same use as $X$-directed.

\section{Arbitrarily Defined}

In addition to the predefined interactions described above, a designer can identify any arbitrary set of interaction coefficients and use them as an interaction. This could be done, for example, in a spreadsheet, and then the results could be copied and pasted into MFDTool.

\section{Constraints}

For each interaction, MFDTool allows the user to identify six types of constraints, which can be mixed and matched as desired. Each constraint can be imposed on any kind of interaction. Although the names of the constraints reflect their common usage, a constraint imposes quite different designs depending on the properties of the interaction.

\section{Global Difficulty}

If one ignores shortcuts and backward links, moving through a hierarchy from top to bottom can be described by a single finite sequence of buttonpresses. A global difficulty constraint acts to assign labels to buttons in a way that minimizes the sum of interaction coefficients along the sequence of buttonpresses for each label. The designer 
specifies the frequencies of search for different pieces of MFD information, and then MFDTool associates page labels with buttons in such a way as to minimize the average difficulty needed to reach information.

\section{Pages to Close Buttons}

Often, labels on a single screen are related to each other, and the designer wants the related page labels to be grouped together on nearby buttons. At other times, labels on different MFD screens are related, and the designer wants those labels to be associated to the same or nearby buttons (e.g., "Cancel" should be in the same place on every page). MFDTool allows the designer to specify as many of these constraints as desired.

\section{Pages to Fixed Buttons}

Sometimes a designer wants to restrict a single label or multiple labels (either on the same screen or on different screens) to a subset of the possible buttons (e.g., always put left engine information on the left side of the MFD screen). MFDTool allows the designer to specify as many of these constraints as desired.

\section{Path Difficulty}

The use of some MFDs requires users to retrieve certain combinations of information. If a user has to check first the status of one system, then the status of a second, and then the status of a third, there will be a path of visited pages that corresponds to this combination of information searches. Moreover, because the system information may be scattered across the MFD hierarchy, designers often include hyperlinks, or shortcuts, to the top of the hierarchy or to other MFD hierarchy locations. MFDTool allows the designer to identify these paths, and then MFDTool optimizes the assignment of page labels to buttons so as to minimize the difficulty of executing these button sequences. MFDTool allows the designer to specify as many of these paths as desired.

\section{Pages to Far Buttons}

Sometimes a designer may want a set of labels to be separated as much as possible. This could be useful in ensuring that a user does not confuse similar labels that are functionally different. It could also help the designer organize labels into different positions on the screen.

\section{Parent-to-Child Variability}

This constraint allows a designer to impose certain types of consistency on the layout design. This constraint attempts to minimize, among specified pages, the variability (standard deviation) in interaction coefficients between the buttons assigned to a page's parent and to itself. For example, if Euclidean distance is used, specified pages will be assigned to buttons so that clicking on the button of a page's parent will then require a move of a constant distance to reach the desired page's button. This would likely provide a consistent motor memory that might make learning and using the MFD easier.

\section{Constraint Costs}

Each constraint has a corresponding numerical cost function that measures how well a constraint is being satisfied by an MFD design. Smaller cost values correspond to better designs, relative to that constraint and its interaction. An optimization algorithm searches through a variety of MFD designs to find one that minimizes (or nearly so) a weighted sum of costs:

$$
\sum_{i} \lambda_{i} C_{i}
$$

where $C_{i}$ is the cost for constraint $i$ and $\lambda_{i}$ is a weighting parameter set by the designer. With the weighting parameter, the designer can emphasize one constraint over another.

\section{Optimization Techniques}

It might seem that with a quantified model of constraints, one could simply let a computer consider all possible mappings of labels to button paths and find the mapping that minimizes constraint cost. Unfortunately, the computational magnitude of the problem precludes such an exhaustive search. Even a small MFD hierarchy with 8 buttons and three levels, for instance, provides 512 unique button paths that reach to the third level. Suppose every label is filled at every level, so that there are eight choices at the top level (one buttonpress), eight choices at each second level (two buttonpresses), and eight choices for each bottom level (three buttonpresses). If all the hierarchical relations between labels are maintained in their button paths, then at each level there are $8 !=40,320$ possible assignments of labels. Thus, the total number of possible mappings that still maintain the hierarchical relations among labels is the cube of 8 ! or approximately $6.55 \times 10^{13}$. As the size of the MFD increases, the number of mappings becomes so enormous that even highspeed computers cannot consider all the possibilities in a timely manner (e.g., MFDs with 20 buttons and five levels are not excessively large in real world applications, but the number of mappings is approximately $8.52 \times 10^{91}$ ). Thus, an approach other than exhaustive trial and error is needed. MFDTool provides two optimization algorithms: hill climbing and simulated annealing (Geman \& Geman, 1984).

In a hill-climbing (or hill-descending, for which only the sign needs to be changed) algorithm, the system is initialized to a particular state (e.g., mapping of labels to buttons), and the cost is calculated for that state. One of the variables of the problem (e.g., a label) is randomly selected and modified (e.g., moved to a new button). A new cost value is calculated, and if the new cost is less than the old cost, the change is kept; otherwise, the change is undone. This process is repeated until the system converges to a state where any change would lead to an increase in cost (e.g., where any change in the mapping would be worse). In complex problems, hill-climbing methods can easily get trapped in a state where any change only increases cost (a local minimum) but the global minimum is very different, with a much smaller cost. To avoid this problem, MFDTool proceeds through many runs of the opti- 
mization with different random initial designs and keeps track of the overall best design. The success of this approach depends on the complexity of the cost space of the system (which is generally unknown). In practice, this algorithm is fast and often ends up with a good optimization.

Simulated annealing is a variation of hill-climbing algorithms that addresses the local minima problem by introducing a controlled way to climb out of local minima and end in a state with the global minimum of cost. Simulated annealing is a stochastic algorithm that at first accepts changes even if they lead to larger costs. As time progresses, a temperature parameter gradually decreases (this is the annealing), so that it becomes less likely that a change leading to an increase in cost will be accepted. As the temperature becomes small, the algorithm becomes essentially hill-climbing. As long as the temperature decreases slowly enough and enough changes are considered at each temperature level, simulated annealing is statistically guaranteed to find the global minimum of a problem. In practice, though, the necessary temperature schedule is too slow, and the number of changes at each level is too large, so simpler approaches are taken that are faster but less certain to find the global minimum.

At the end of either optimization process, the system should be in a state with a low (but perhaps not optimal) cost. Being certain of finding the true optimal state with the absolute lowest possible cost would be prohibitive because of the complexity of the search problem. Having a system in a state with low cost corresponds to having an MFD design that satisfies the imposed constraints.

In theory, simulated annealing should give a better optimized result than does the hill climbing algorithm. However, in practice, simulated annealing's performance depends on the parameters selected, so in many instances the hill climbing procedure is preferable. In MFDTool, a designer can just use the default optimization parameters and likely get fairly good optimization results, or a designer can set a variety of optimization parameters to finetune the process of searching for the MFD design that best satisfies the constraints.

\section{An Example of MFD Design}

To demonstrate the capabilities of MFDTool, consider the design of a relatively simple system for entering alphabet characters. The qwerty keyboard is the standard for two-handed keying of information. For other systems, such as palm pilots and one-handed keyboards in aircraft, there is no established standard. The default design tends to be to order the letters alphabetically. This is probably suboptimal, since some letters are used more frequently than others and some letter strings are used more often than other letter strings. A hard-coded keyboard is an MFD with only one function assigned to each key, and MFDTool can be applied to the design of such a system. Examples of optimized designs for more complicated MFDs are available with the MFDTool program and are discussed in the user's guide.
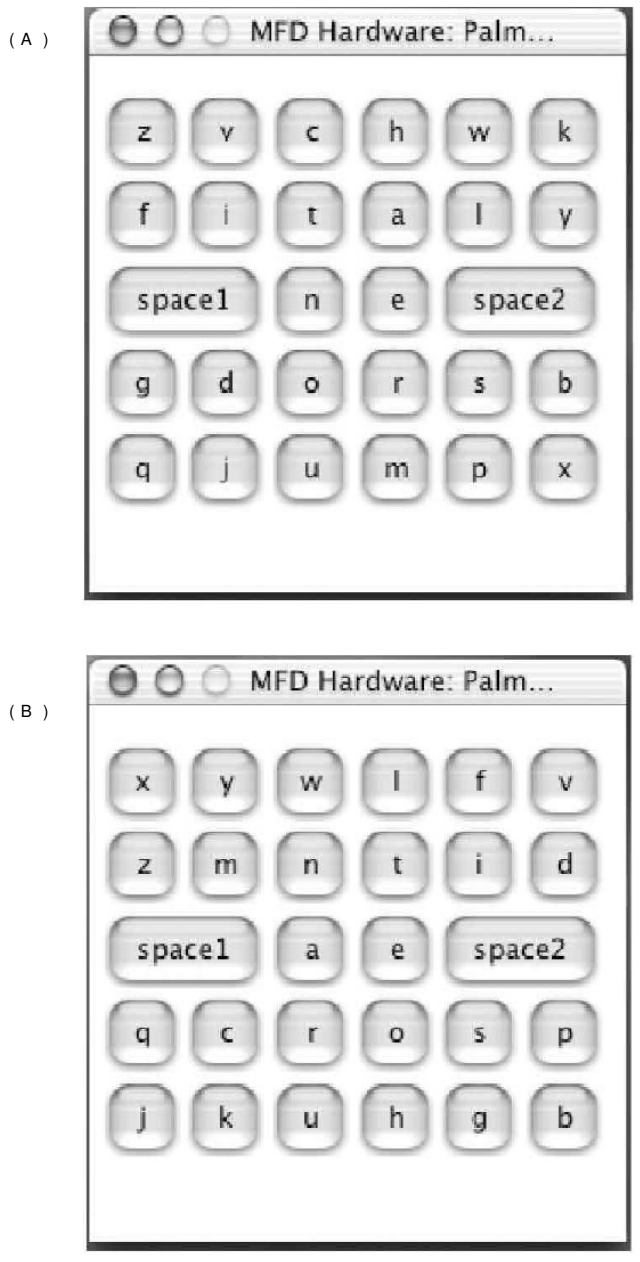

Figure 2. Two designs for one-finger keyboard entry. (A) The original fitaly keyboard for one-finger use. This design was produced by Textware Solutions. (B) The optimal one-finger keyboard designed by MFDTool with the fitaly hardware design.

There has recently been interest in the design of one-finger keyboards. Textware Solutions (http://www. twsolutions.com) recently proposed the fitaly keyboard, which they claimed was able to increase typing by one finger/pen to a speed almost as fast as a qwerty twohanded keyboard. A schematic of the fitaly keyboard is shown in Figure 2A. (All screen shots are taken from the MFDTool program running on Mac OS X.)

The engineers at Textware Solutions based their design on consideration of the frequency with which individual letters were used and the frequency of letter-to-letter transitions. The former frequencies are published on their Web site and were used to create an analogous design in MFDTool. Since users of such a keyboard would soon memorize where every letter is on the keyboard, the layout of letters should be such that movements are minimized. A Fitts's law interaction was used. The fitaly keyboard includes two larger than usual keys for spaces, and the 

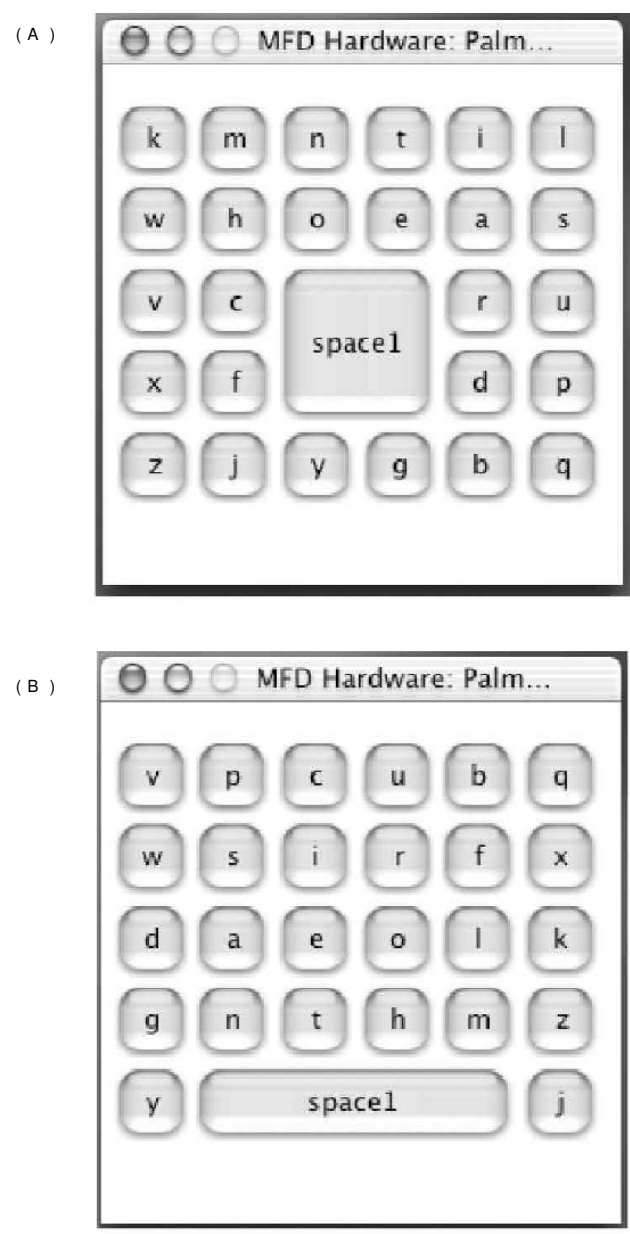

Figure 3. Optimized one-finger keyboards for different physical designs. (A) A variation on the fitaly design, with one space button near the middle of the keyboard. (B) A variation of the $f i$ taly design with the space buttons merged and placed on the bottom row. This optimized design results in the smallest average movement time to strike a key.

MFDTool design was similarly restricted so that these keys could be used only for space characters. Significantly, MFDTool creates a design, shown in Figure 2B, quite similar to the fitaly design. In particular, the six letters in the middle three rows of the middle two columns are the same in both designs. These correspond to the most frequently used letters.

The discrepancies between the two designs probably correspond to the effect of letter-to-letter transitions, which the current MFDTool example does not consider (but could be implemented if the data were available). Without consideration of those transitions, the MFDTool design is slightly more optimized relative to the global difficulty constraint. The MFDTool design has a cost of $182.77 \mathrm{msec}$, whereas the fitaly design has a cost of $185.85 \mathrm{msec}$. The costs refer to the average time (in milliseconds) to strike a key from the start position (the "e" key).
MFDTool can also be used to consider optimization of other hardware designs. For example, in Figure 3A, the two space buttons are merged into a single large button and placed slightly off the center of the screen; the optimized assignment has a larger cost $(184.28 \mathrm{msec})$ than does the optimized layout for the hardware used by the original fitaly system.

Better results are found for the design in Figure 3B, where the space button is one row high and four columns wide and placed on the middle bottom of the display. The cost for the optimized design is $167.61 \mathrm{msec}$, which is $13.74 \mathrm{msec}$ faster than any other design discussed so far. Relative to the original fitaly layout, this design has an 18.24-msec advantage.

Of course, these alternatives to the original fitaly design may not be as good as the original, if the transition probabilities between letters are also considered. Also, one would want to experimentally validate the predicted differences in the designs.

Perhaps an even better approach for the use of MFDTool would be to identify the specific text that must be entered for particular uses of the keyboard. With such data, a designer could create a set of path difficulty constraints that corresponds exactly to that text. Such an arrangement will automatically include the relevant statistics on frequency of letter use and will include statistics for higher order combinations of letter strings.

For example, suppose a person planned to frequently type the name francis on a one-finger keyboard. Using the design in Figure 3B, it would take approximately $1,227 \mathrm{msec}$ to move from from the "f" key to the "r" key to the "a" key and so on to the "s" key. However, it is possible to ask MFDTool to produce a one-finger keyboard design that, in addition to doing the optimization used before, also minimizes the time required to type francis. Such a design is shown in Figure 4. Note that the keys for each sequen-

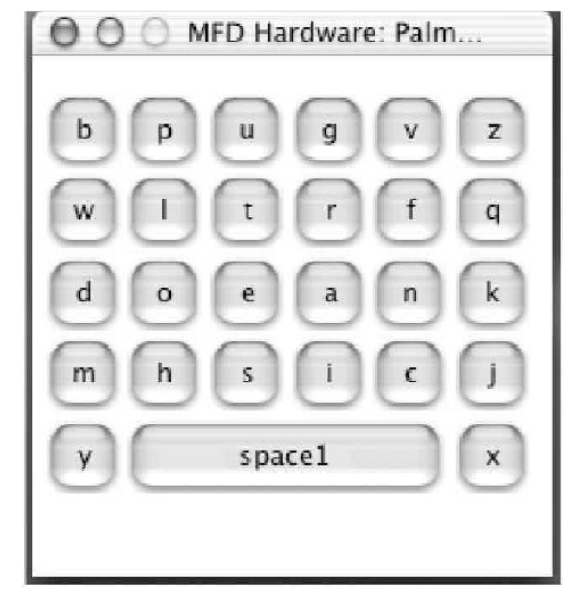

Figure 4. An optimized one-finger keyboard that has been designed to minimize the time required to press the sequence of buttons spelling francis. Notice that the pairs of letters in this sequence are on keys that are next to each other. 
tial pair of letters are directly next to each other. This minimizes the time required to move between buttons when typing francis. The predicted movement time for typing francis is $1,007 \mathrm{msec}$, a savings of $220 \mathrm{msec}$, as compared to the design in Figure 3B. One could add many more sequences of letters and ask MFDTool to produce the optimal design for all of them.

Of course, there is a tradeoff in the constraints. By decreasing the time required to make the movements to type out francis, MFDTool had to slightly increase the average time required to strike any key. The predicted average time to strike a key for the design in Figure 4 is $168.8 \mathrm{msec}$, which is about $1 \mathrm{msec}$ longer than that for the design in Figure 3B. Clearly, the validity of the optimized design depends on the validity of the interactions and constraints at identifying those aspects of the human-computer interface that are truly important.

\section{Conclusions}

The optimization process used by MFDTool can produce good designs only if the interactions and constraints imposed by the designer accurately capture the important aspects of the human-computer interaction. Naturally, if a designer imposes inappropriate constraints, the resulting "optimized" design will not be a good one.

Thus, the ability of MFDTool to produce good designs depends on the ability of designers to identify the important characteristics of the human-computer interaction for a particular task. Of course, if designers cannot identify the characteristics of the human-computer interaction, it is unlikely that any design method will produce a good design. MFDTool's additional requirement is that the designer be able to quantify those characteristics by defining interactions and constraints. It remains an open question whether this additional requirement substantially limits the applicability of MFDTool. As is the case for every design approach, the only way to be sure a system design meets the needs of the user(s) is to experimentally validate the system.

MFDTool should be useful to two groups of researchers. First, it should be useful for designers who need to build working MFDs. MFDTool is flexible enough to work with a variety of design situations and, given the proper information, should be able to greatly speed up and improve the design of MFDs.

Second, MFDTool should be useful to academics who want to study the details of human-computer interactions. Many of the guidelines that are currently used by designers for MFD design have not been rigorously tested, because it has never been clear how to test them. Even for cases in which a guideline has been tested (Francis, 2000; Vandierendonck, Van Hoe, \& De Soete, 1988) it is not obvious that the conclusions hold in other situations, nor is it obvious which guidelines are most important. Running such tests has always been difficult, because it is not a triv- ial task to build an MFD that satisfies various guidelines. MFDTool now provides the ability to produce a large variety of MFDs, each optimized to emphasize different guidelines. These different MFD designs can now be compared with each other (with standard experimental approaches), so that researchers can determine which, if any, of the guidelines accurately capture important traits of MFD use.

\section{REFERENCES}

Calhoun, G. (1978). Control logic design criteria for multifunction switching devices. Proceedings of the Human Factors Society 22nd Annual Meeting (pp. 383-387). Santa Monica, CA: Human Factors Society.

Cook, R., \& Woods, D. (1996). Adapting to new technology in the operating room. Human Factors, 38, 593-613.

Cuomo, D. L., Borghesani, L., Khan, K., \& Violett, D. (1998). Navigating the company Web. Ergonomics in Design, 6, 7-14.

DEPARTMENT OF DEFENSE (1981). Military standard: Human engineering design criteria for military systems, equipment, and facilities (Rep. No. MIL-STD-1472D). Washington, DC: Author.

Fisher, D. L., YungKurth, E., \& Moss, S. (1990). Optimal menu hierarchy design: Syntax and semantics. Human Factors, 32, 665-683.

FitTs, P. M. (1954). The information capacity of the human motor system in controlling the amplitude of movement. Journal of Experimental Psychology, 47, 381-391.

FrancIS, G. (2000). Designing multifunction displays: An optimization approach. International Journal of Cognitive Ergonomics, 4, 107-124.

Geman, S., \& GEMAN, D. (1984). Stochastic relaxation, Gibbs distributions, and the Bayesian restoration of images. IEEE Transactions on Pattern Analysis \& Machine Intelligence, 6, 721-741.

Holley, C., \& Busbridge, M. (1995). Evolution of the Venom variant of the AH-1W super-cockpit. Proceedings of the American Helicopter Society 51 st Annual Forum (pp. 1436-1449). Fort Worth, TX.

LiND, J. (1981).Evaluation of cockpit procedures, displays, and controls for stores management in the advanced aircraft armament system (AAAS) (Naval Weapons Center Technical Memorandum No. 4538). China Lake, CA: Naval Weapons Center.

Obradovich, J. H., \& Woods, D. D. (1996). Users as designers: How people cope with poor HCI design in computer-based medical devices. Human Factors, 38, 574-592.

Reardon, M. J., \& Francis, G. (1999). Reducing the risk of aviatormultifunction display interface problems with human factor models and optimization design methods. SAFE Journal, 29, 100-106.

REISING, J., \& CURrY, D. (1987). A comparison of voice and multifunction controls: Logic design is the key. Ergonomics, 30, 1063-1077.

Rogers, W., Cabrera, E., Walker, N., Gilbert, K., \& Fisk, A. (1996). A survey of automatic teller machine usage across the adult life span. Human Factors, 38, 156-166.

Sirevaag,E., Kramer, A., Wickens, C., Reisweber, M., Strayer, D., \& GRENELL, J. (1993). Assessment of pilot performance and mental workload in rotary wing aircraft. Ergonomics, 33, 1121-1140.

SPIGER, R, \& FARRELL, R. (1982). Survey of multi-function display and control technology (Report No. NASA-CR-167510). Washington, DC: National Aeronautics and Space Administration.

Vandierendonck, A., Van Hoe, R., \& De Soete, G. (1988). Menu search as a function of menu organization, categorization and experience. Acta Psychologica, 69, 231-248.

Williges, R., Williges, B., \& Fainter, R. (1988). Software interfaces for aviation systems. In E. Wiener \& D. Nagel (Eds.), Human factors in aviation (pp. 463-491). San Diego: Academic Press.

(Manuscript received November 14, 2002; revision accepted for publication January 15,2003 .) 